\title{
Aspirine et hémorragie digestive
}

Peu de medicaments ont eu une fortune aussi brillante et durable que Гaspirine, introduite en thérapeutique par Dreser en 1889; le derive acétylé de Гacide salicylique présente en effet un gout moins désagré-able que le sel sodique (plus actif), et on espérait que son insolubilité relative en milieu acide éviterait $\Gamma$ irritation de la rnuqueuse gastrique, habituelle avec les autres salicylates. Lors de la découverte des effets miraculeux de la cortisone sur les rhumatismes inflammatoires, la designation humoristique du nouveau produit sous le nom d'« aspirine du riche» était encore un hommage indirect rendu à Tinfluence sedative remarquable des salicylés sur les arthropathies; ils ne perdirent d'ailleurs pas de terrain pour autant, puisqu'on leur attribue mainte-nant une activité pharmacologique «cortisone-like», c'est-à-dire par le relai de Гhormone corticotrope (ACTH) hypophysaire.

Un medicament aussi répandu ne pouvait manquer d'engendrer des reactions d'intolérance: les dangers d'acidose sont classiques aux fortes doses prescrites naguère dans la maladie de Bouillaud; des crises allergiques (asthme, urticaire, ædème de Quincke) surviennent chez les individus sensibilisés; les hémorragies digestives, pour avoir

\section{Editorial}

275

été dépistées en date relativement récente, ne doivent pas être mini-misées, la relation entre Taccident d'allure dramatique et $\Gamma$ ingestion d'une drogue de reputation si inoffensive, échappant le plus souvent aux intéressés.

II y a une vingtaine d'années qu'à Londres, A. H. Douthwaíte et G. A. Líntott d'une part, en gastroscopie, Sir Arthur Hurst d'autre part, attirèrent pour la premiere fois Гattention - sans grand succès, semble-t-il - sur la responsabilité de l'aspirine dans le déterminisme des lesions gastriques; les gastro-entérologues commencèrent à s'in-quiéter un peu plus quand on invoqua la similitude d'action entre aspirine et cortico-stéroïdes, et les enquêtes récentes fournissent des chiffres impressionnants. En moins de 3 ans, M. Levrat et R. Lambert (Presse médicale du 10 dec. 1958, p. 1945 à 1949) ont recueilli dans leur service hospitalier de Lyon pas moins de 52 observations d'acci-dents digestifs déclenchés par l'aspirine, dont les $3 / 4$ avec hémorragíe! Le déterminisme des lesions irritatives de la muqueuse gastrique a été observe par gastroscopie, par gastro-biopsie ou sur pieces de gastrectomie; des ulcères anciens peuvent être reveilles. La réalité de Гinfluence hémorragipare de l'aspirine a été démontrée, avec toutes les precisions de la statistique, par Lange, d'Oslo (Gastroenterology; vol. 33, pp. 770-788, nov. 1958): prescrivant de l'aspirine pendant 12 à 23 jours à 110 malades divers, et pratiquant la reaction de la benzidine dans leurs selles, il constate un rapport: Benzidine negative/ Benzidine positive de 1 à 7 , avant; de 1 à 1,6 , pendant, et de 1 à 2,8 , après le traitement.

L'aspirine peut faire saigner des ulcéreux connus antérieurement ou non, mais prouvés radiologiquement; le medicament serait même responsable, dans certaines series, de plus des deux tiers des hémor-ragies. Chez Levrat et Lambert, on relève 15 hémorragies ulcéreuses 
certaines contre 21 sans substratum organique precis, une ulcération aiguë superficielle n'étant naturellement pas exclue pour autant. Le mémoire très complet des médecins lyonnais se termine par les con-seils d'ínterroger systématíquement les malades qui saignent de Гesto-mac sur Tusage antéríeur, habítuel oufortuít, d'aspirine; et de défendre en permanence les salicylés aux ulcéreux.

*

L'aspirine n'est pas dangereuse seulement au niveau du tube digestif supérieur: elle peut être aussi responsable en partie de certaines 